\title{
Comparison analysis of travel demand forecasting model
}

\author{
LIU Wen Zhi ${ }^{1,2, a}$, LU Hua $\mathrm{Pu}^{1, \mathrm{~b}}$ \\ ${ }^{1}$ Institute of Transportation Engineering, Tsinghua University, Beijing, 100084, P.R. China \\ ${ }^{2}$ School of Management, Beijing Union University, Beijing, 100101, P.R. China \\ aliuwenzhi@buu.edu.cn, ${ }^{b}$ luhp@mail.tsinghua.edu.cn
}

\begin{abstract}
Keywords: Transportation planning; Travel demand; Forecasting; Model
Abstract. Reasonable transportation planning is a fundamental solution of traffic jams in big cities and the core is travel demand forecast. Reasonable travel demand forecast will provide scientific evidence for decision of transportation planning. Thus, it seems very urgent to do some research on travel demand forecast in varies countries. In this paper, we first summarize most of research work on the travel demand forecasting model in domestic and overseas. Then, discuss the future work in this field.
\end{abstract}

\section{Introduction of Aggregated Model}

In the early 60's of last century, 《Chicago Area Transportation Study》 contributed to the formulation and development of transportation planning theory and method. This law pointed out that urban transportation planning should be based on traffic survey to obtain the financial subsidies of highway construction from federal governments. The earliest travel demand forecasting model was simple and aggregated model, contains several parameters, and using aggregated data to estimate them. The representation of aggregated model is "four stage model", which is formulated in the 70's of last century. The model contains trip generation, distribution, mode split and traffic assignment. After that, many scholars have inducted the theory of other disciplines to made corresponding improvement to four stage model. Considering to the data collection, all those improved models still takes traffic district as a basic analysis unit[1]。Thus, the defects of traditional aggregated model are as follows: 1) lack of economic theory base, social, Psychology and behavior analysis. 2) lack of consistency between each stage, no feedback mechanism. 3) lack of statistical method 4) lack of evaluation mechanism between transportation management and demand 5) forecasting ability is relatively weak.

Considering on the above defects of four stage model, scholars have studied and developed more complex model which is disaggregated and more fit with real demand. This type of model is based on the theory of probability, economics, sociology and behavioral science, and accurately describe the selection process of person and household travel[2]. The representation of disaggregated model is Logit model which is deducted from Luce in 1959. After that, McFadden who is from MIT has discussed the logit model and it's features, then formed the theoretical system, put them into the practical stage in 1974[3]. In the last 70's of last century, the basic idea and methods of disaggregated model were applied to the actual forecast and be improved in 80 's. Since then, along with the development of computer technology and simulation algorithm, there have been some more complicated and abundant disaggregated models, such as Probit model, Mixed Logit model, OGEV model (Ordered generalized extreme value), PCL model (Paired combinatorial logit), MNP model

(Multinomial Probit) and CNL model（Cross-nested logit) and so on[4].

\section{Disaggregated Model}

In the end of 1970's, activity based travel demand forecasting disaggregated model was formed by Chapin, Hagerstrand, Fried and Traffic research group of University of Oxford. Their method advocated that personal activities are influenced by time and space constrains. People's social and economic attributes and the potential needs will together give effect on the choices of activity type and participation mode. But the common point of research during this period is deep understanding of 
travel behavior, not to establish a forecasting model. In terms of travel demand forecasting, the traditional four stage model is main method in our countries currently. In terms of disaggregated model, most of domestic researches focused on the basic theory of NL, MNL, Mixed Logit and Probit model, that is, the utility maximization theory and modeling and calibration parameters. The application area of disaggregated model included travel mode selection, public transport fare formulation, travel route choice, parking demand management, market share of transportation product forecast, route traffic travel mode share forecast, etc. Generally speaking, the domestic research on the individual travel behavior analysis and travel demand forecasting model based on activity method is carried out late, and the researches are not much. A small number of scholars, such as Lu Huapu, Juan Zhi Cai, Huang Haijun, Li Zhiyao, have done part of research from the morning and evening commute activity, flextime, travel chain, the resident's daily activities arrangement model, time selection and the mode choice model, sensitivity analysis of the model.

\subsection{Individual activity / travel plan generating and scheduling model}

Individual activity / travel plan generating and scheduling model is the core content of behavior theory. Different behavior characteristics produce different forms of activities and travels. The travel demand forecasting model based on the theory of activity behavior is dedicated to simulate the activities and travel characteristics of people, including the types of activities, the starting time, duration, frequency, location and traffic mode. Bradley and Axhausen put forward a series of questions list, through answering questions, in-depth understanding of Walker's social and economic attributes of the impact of travel characteristics [6]. Bowman develops the daily activity planning model, and takes the Portland city residents travel survey data as an example, and carries on the application and the appraisal to the model [7].

The construction methods of individual activity / travel plan generating and scheduling model mainly include econometrics selection method and calculation process model. The former one mainly uses the utility maximization theory, such as MNL, NL, etc. the latter one is a rule based simulation model. The representation included AMOS which is developed by RDC in 1992, SCHEDULER framework which is formed by Gärling in 1998 and ALBATROSS which is muti-agent system developed by Arentze and Timmermans in 2000. Their structures are as shown in the following Figure 1, Figure 2 and Figure3. The common point is these three models are all generate the activity travel plan. Where, SCHEDULER and ALBATROSS are more similar, and both are lack of assessment function on the activity/ travel plan when they are comparing with AMOS. The first two models are based on heuristic path, and the process of activity selection is simulated by the simulator. The difference is that SCHEDULER uses the rule based method, while ALBATROSS uses the decision tree method.

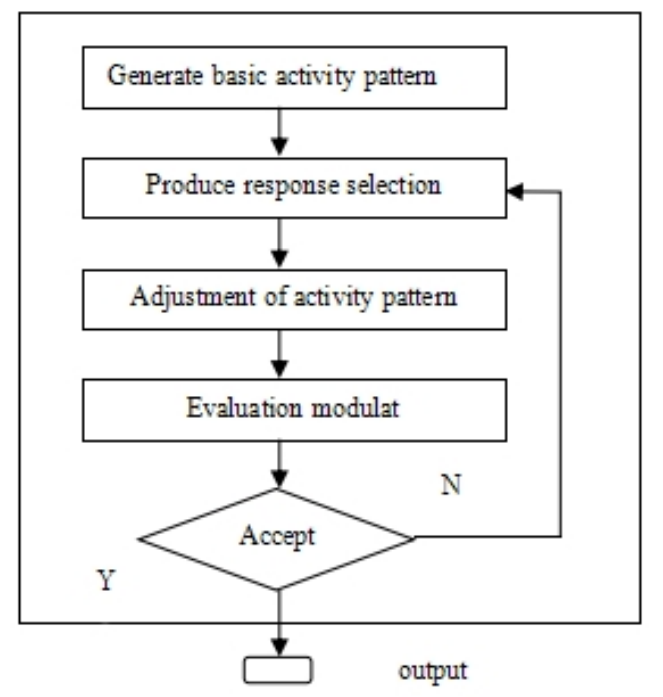

Fig.1 AMOS structure 


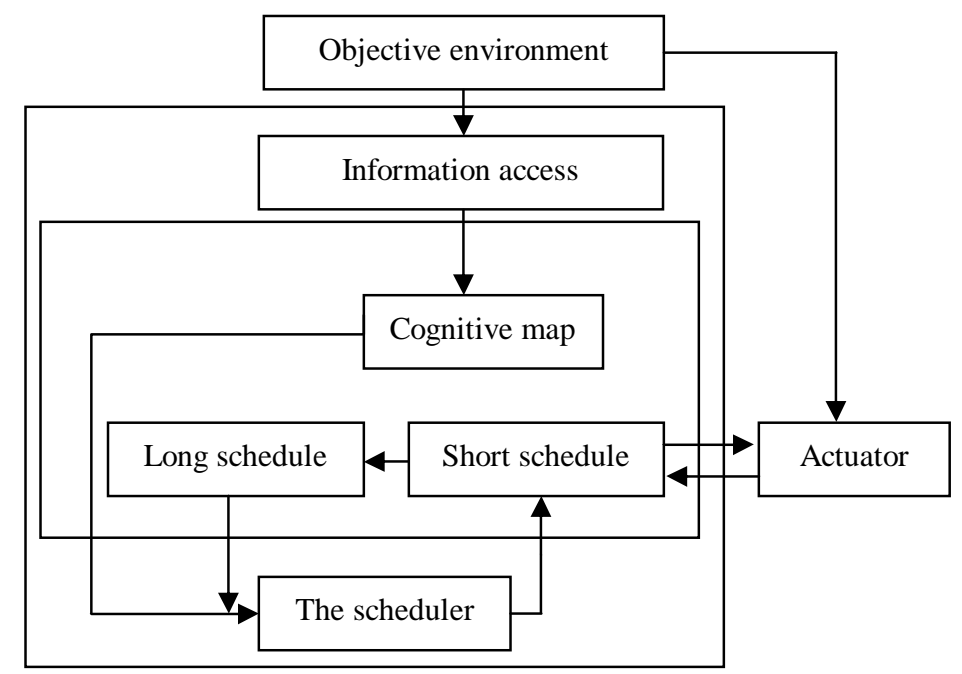

Fig.2 SCHEDULER structure

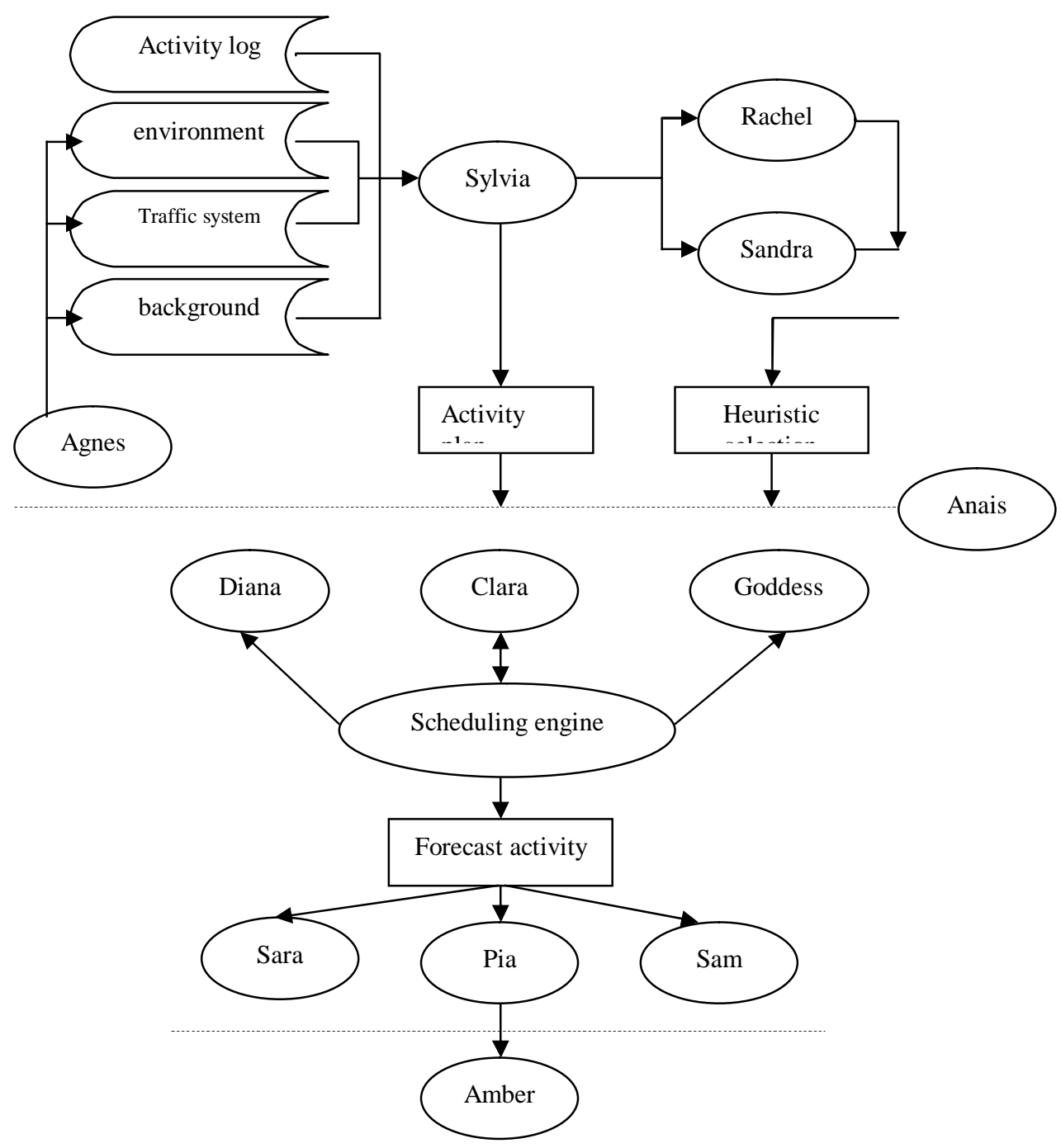

Fig.3 ALBATROSS structure

\subsection{Mode choice model}

Travel mode choice plays an important role in traffic management and control. Many scholars in the world have got remarkable achievements on this topic. To sum up, most of the researches used the traditional four stage model and the theory of random utility maximization, and pointed out that travel 
mode choice is the individual behavior. Therefore, Thoes researches are lack of description of the behavior characteristics of travel mode choice, and the accuracy of the model is also insufficient. In fact, travel mode choice is related to the entire trip chain. For example, when you go out with a car or a bike, you usually choose to drive or ride a bike to back home at the end. Based on this kind of assumption, Ben-Akiva and other scholars were pointed out mode choice model under the restriction of trip train in the end of 1990's and then has been widely used[8]. The representative areas are included Holland, Sweden, Denmark, Italy, New York, Ohio, Boston, San Francisco , Portland and other regions. However, travel mode choice is not only related to the whole trip chain, but also influenced by the number of family cars as well as the car allocation plan.

Generally speaking, the defects of activity based trip character analysis and travel demand forecasting model are as follows:

1) The methods of model construction are mainly including MNL and NL, and their parameter estimation process is more complex and larger calculation.

2) In terms of model structure, the connection between each sub models is still very low, and relatively isolated.

3) Most of researches are focused on the individual level of travelers, lack of research on household activity decision and task allocation level.

\section{Research prospects}

Research on the activity based travel demand forecasting disaggregated model has a very important theoretical value and practical meaning for deep understanding and intuitive analysis on residential activity, travel behavior and interaction type, for the improvement and development of transportation planning model, for formulation, analysis and evaluation of travel demand management policies and measures. For the application of model, we can forecast and analyze the changes of travel demand under different transportation policies and land use characteristics. For example, relocation, expansion and construction of large institutions, road and subway, flexible arrangements of work time etc. For those above situation, we can forecast travel demand changes under different policies and measures, according to the changes of residential, work and school location, road net structure, trip time. Currently, research in this field is still in the primary stage in our country, and reasons are mainly in the following three aspects: at first, there is a certain degree of difficulty in obtaining Municipal road network graph. This will affect traffic flow assignment. Furthermore, affect the construction of model application and dynamic feedback function, such as travel time feedback, travel cost feedback and so on. Second, Model lime selection and variable selection should be combination with the basic economic level and traffic conditions of the city. At last, the methods should be developed from the simple utility maximization theory to the rule based calculation process model. By the information access tools, channels and capacity constraints, it is impossible to compare, weigh all travel information to calculate the maximum utilities to make the best judgment. Therefore, it should be based on certain decision rules to select the plans which are actual and suitable for individual and household status.

Due to the above reasons, most of the research work in this field is just limited on the theoretical model construction, and it is difficult to put into practical application. Therefore, the in-depth study and research on the activity and household decision based travel demand forecasting problem will greatly promote the development of traffic planning theory and related disciplines.

\section{Acknowledgements}

This work was financially supported by the Beijing outstanding talent training Project (20140000204400001) and A new starting point plan project of Beijing Union University (Sk10201503). 


\section{References}

[1] Messenger Todd, Ewing Reid. Transit oriented development in the Sun Belt [J]. Transportation Research Record, 1996, 1552:145-153.

[2] Cervero R, Carolyn R. Travel choices in pedestrian versus automobile oriented neighborhoods [J]. Transport Policy, 1996, 3(3): 127-141.

[3] Farooq B, Miller E J. Towards integrated land use and transportation: A dynamic disequilibrium based microsimulation framework for built space markets [J]. Transportation Research A, 2012, 46(7): 1030-1053.

[4] Rashidi, Taha Hossein. A behavioral housing search model [J]. Transportation Research A, 2012, 46(7): 1097-1107.

[5] Lee Brian H Y, Waddell Paul. Residential mobility and location choice[J]. Transportation, 2010, 37(4): 587-601.

[6] Doherty, S.T., E.J. Miller. A Computerized Household Activity Scheduling Survey[J]. Transportation, 2001, 27(1): 75-97.

[7] Habibian M, Kermanshah M. Exploring the role of transportation demand management policies' interactions[J]. Scientia Iranica, 2012, 18(5): 1037-1044.

[8] Ben-Akiva, M., J. Bowman, S. Ramming et al. Behavioral realism in urban transportation planning models.In: Transportation Models in the Policy-Making Process A: Symposium in Memory of Greig Harvey. Asilomar Conference Center, California, 1998. 